\title{
Hypoxia and aging
}

\author{
Eui-Ju Yeo ${ }^{1}$
}

\begin{abstract}
Eukaryotic cells require sufficient oxygen $\left(\mathrm{O}_{2}\right)$ for biological activity and survival. When the oxygen demand exceeds its supply, the oxygen levels in local tissues or the whole body decrease (termed hypoxia), leading to a metabolic crisis, threatening physiological functions and viability. Therefore, eukaryotes have developed an efficient and rapid oxygen sensing system: hypoxia-inducible factors (HIFs). The hypoxic responses are controlled by HIFs, which induce the expression of several adaptive genes to increase the oxygen supply and support anaerobic ATP generation in eukaryotic cells. Hypoxia also contributes to a functional decline during the aging process. In this review, we focus on the molecular mechanisms regulating HIF-1a and aging-associated signaling proteins, such as sirtuins, AMP-activated protein kinase, mechanistic target of rapamycin complex 1, UNC-51-like kinase 1, and nuclear factor $\mathrm{kB}$, and their roles in aging and aging-related diseases. In addition, the effects of prenatal hypoxia and obstructive sleep apnea (OSA)induced intermittent hypoxia have been reviewed due to their involvement in the progression and severity of many diseases, including cancer and other aging-related diseases. The pathophysiological consequences and clinical manifestations of prenatal hypoxia and OSA-induced chronic intermittent hypoxia are discussed in detail.
\end{abstract}

\section{Introduction}

Oxygen $\left(\mathrm{O}_{2}\right)$ plays critical roles in aerobic respiration and metabolism as the final electron acceptor of the mitochondrial electron transport chain, which is responsible for generating the majority of ATP within an eukaryotic cell ${ }^{1}$. Therefore, an adequate concentration of oxygen is required by eukaryotic cells to maintain a variety of biological activities and ensure survival. When the oxygen demand exceeds its supply, oxygen levels in the whole body or local tissues decrease (termed hypoxia), leading to a metabolic crisis, threatening physiological functions and viability. Due to the critical roles of oxygen in respiration, metabolism, and survival, eukaryotes have developed an efficient and rapid oxygen sensing system ${ }^{1}$.

A change in the oxygen concentration in the surrounding atmosphere is detected by central and arterial chemoreceptors $^{2}$. Central chemoreceptors are present in the medulla of the brainstem near the respiratory centers. Arterial chemoreceptors are present in the aortic body and carotid body. The activation of arterial

\footnotetext{
Correspondence: Eui-Ju Yeo (euiju@gachon.ac.kr)

${ }^{1}$ Department of Biochemistry, College of Medicine, Gachon University, Incheon 21999, Republic of Korea
}

chemoreceptors stimulates the neurotransmitter release pathway $^{3}$ and modulates the activity of a neutral endopeptidase, neprilysin (NEP), which modifies the cellular response to hypoxia by hydrolyzing substance $\mathrm{P}^{4}$. Oxygen sensing is also controlled by pulmonary neuroendocrine cells in neuroepithelial bodies, exerting chemosensitivity, which is important for oxygen sensing in early developmental stages of life ${ }^{5}$. These stimulated chemoreceptors increase sympathetic neural activity (SNA) and the arterial pulmonary and systemic blood supply to obtain more oxygen ${ }^{6}$. On the other hand, the expression of several adaptive genes is induced to increase the oxygen supply and support anaerobic ATP generation in eukaryotic cells. These hypoxic responses are controlled by hypoxia-inducible factors (HIFs) ${ }^{7,8}$. Over the past two decades, various biochemical and genetic studies have expanded our knowledge of hypoxia to the cellular and molecular levels. Therefore, the HIF pathway is briefly summarized in the first section "Hypoxia and the HIF pathway".

In addition, hypoxia potentially contributes to functional decline during the aging process. HIF pathways cross-talk with the sirtuins, AMP-activated protein kinase 
(AMPK), mechanistic target of rapamycin complex 1 (mTORC1), UNC-51-like kinase 1 (ULK1), and nuclear factor $\kappa B(N F \kappa B)$ pathways in hypoxia and aging ${ }^{9-12}$. The putative molecular mechanisms underlying the effects of hypoxia, including HIF- $1 \alpha$, AMPK, sirtuins, mTORC1, ULK1, and NFKB, are discussed in the section "HIF- $1 \alpha$ and aging".

Traditionally, physiological research has focused on the effects of acute and chronic sustained hypoxia and the human adaptive response to high altitude. However, in recent years, the effects of obstructive sleep apnea (OSA)induced intermittent hypoxia and prenatal hypoxia have garnered interest due to the accelerated progression and severity of many diseases, including cardiovascular and metabolic diseases, neurological disorders (including depression and neurodegenerative diseases), cancer, and aging. Therefore, the pathophysiological consequences and clinical manifestations of OSA-induced chronic intermittent hypoxia and prenatal hypoxia are discussed here in detail.

\section{Hypoxia and the HIF pathway \\ Regulation of HIF-1a expression and activity during hypoxia}

Because of its involvement in a diverse range of disease states, the mechanism by which cells sense hypoxia and transduce a signal to the HIF pathway has been intensively investigated by many researchers. Members of the human HIF family (HIF-1, HIF-2, and HIF-3) are heterodimeric transcription factors, each of which is composed of an $\alpha$ and a $\beta$ subunit (also termed as aryl hydrocarbon receptor nuclear translocator; ARNT) ${ }^{13,14}$. Cellular and developmental responses to hypoxia are mainly mediated by HIF$1 \alpha$, which is encoded by the HIF1A gene ${ }^{13,15}$. Three HIF$1 \alpha$ isoforms have been identified (HIF- $1 \alpha$, HIF- $2 \alpha$, HIF$3 \alpha$ ), which are generated by alternative splicing. Among the three isoforms, isoform 1 has been extensively studied and characterized, both structurally and functionally ${ }^{16}$. The dysregulation and overexpression of HIF1A induced by genetic alternations and hypoxia have been implicated in a number of pathophysiologies, including ischemia and cancer ${ }^{17}$. HIF-1 $\alpha$ contains a basic helix-loop-helix domain, two distinct PAS (PER-ARNT-SIM) domains, a nuclear localization signal motif, two (carboxy-terminal and amino-terminal) transactivating domains (CTAD and NTAD, respectively), and an inhibitory domain that represses the transcriptional activities of CTAD and $\mathrm{NTAD}^{18}$. In contrast to HIF- $1 \alpha$, which is upregulated under hypoxia, HIF- $1 \beta$ is expressed constitutively.

HIF-1 responds to systemic oxygen levels by interacting with hypoxia responsive elements (HRE) located in the promoter or enhancer regions of hypoxia-responsive genes to induce transcription ${ }^{19-24}$. HIF-1 induces the transcription of more than 100 genes $^{25,26}$ involved in various biological processes, such as angiogenesis, erythropoiesis, anaerobic glycolytic metabolism, $\mathrm{pH}$ regulation, cell proliferation and survival, inflammation and immunity, and cancer metastasis (Table 1). HIF-1 induces the expression of several pro-angiogenic factors, including vascular endothelial growth factor (VEGF), which promotes the formation of new blood vessels and oxygen delivery to hypoxic regions ${ }^{17,24,27,28}$. In addition, HIF-1 increases oxygen transport by enhancing erythropoiesis through the upregulation of erythropoietin (EPO). HIF-1 also stimulates the uptake and anaerobic glycolysis of glucose by increasing the expression of glucose transporters, such as GLUT1 and GLUT3, and several glycolytic enzymes, including hexokinases. HIF-1 activation increases the conversion of pyruvate to lactate by upregulating pyruvate kinase and lactate dehydrogenase. HIF1 promotes lactic acid efflux by monocarboxylate transporter and the conversion of $\mathrm{CO}_{2}$ to carbonic acid $\left(\mathrm{H}_{2} \mathrm{CO}_{3}\right)$ by carbonic anhydrase to counteract intracellular acidosis. Furthermore, HIF-1 affects cell proliferation and survival by modulating the expression of CMYC, insulin-like growth factor 2 and other components of the cell cycle and death pathways. HIF-1 also induces inflammation and immunity by upregulating tumor necrosis factor $\alpha$ (TNF $\alpha)$ and cancer metastasis by upregulating fibronectin 1 . The functions of HIF-1 itself and HIF-1 target genes have been effectively examined using siRNA knockdown-based validation ${ }^{29}$.

A variety of factors are implicated in the mechanism regulating HIF- $1 \alpha$ expression and activity ${ }^{25}$. HIF- $1 \alpha$ expression is controlled at the levels of transcription and translation. In addition, several factors regulate the stability and activity of HIF- $1 \alpha$ at the post-translational level in an oxygen-dependent or oxygen-independent manner. The key factors that regulate its abundance and activity are summarized in Table 2 .

Although the HIF1A gene is constitutively expressed at low levels under normoxic conditions, it is often significantly upregulated in response to hypoxia ${ }^{30-35}$. The transcriptional modulators of HIF-1 $\alpha$ expression include transcription factors, such as nuclear factor kappa B $(\mathrm{NF} \kappa \mathrm{B})$ and signal transducer and activator of transcription 3 (STAT3), and coactivators, such as p300/ CREB binding protein $(\mathrm{CBP})^{36,37}$. The expression of the HIF1A gene may also be upregulated by reactive oxygen species (ROS) through the redox-sensitive transcription factor, NF- $\mathrm{kB}^{38}$. Vanillin possesses anti-metastatic and anti-cancer activities and inhibits STAT3-mediated HIF$1 \alpha$ mRNA expression in human malignant melanoma cells $^{39}$. Similarly, YC-1, 3-(5-hydroxymethy-2-furyl)-1benzylindazole, functions as a potential anticancer drug targeting HIF- $1^{40}$, presumably by inhibiting NFKBmediated and STAT3-mediated transcription of the HIF1A gene. 
Table 1 Representative HIF-1 target gene products

\begin{tabular}{|c|c|c|}
\hline Functional category & Symbol & Full name \\
\hline \multirow[t]{6}{*}{ Angiogenesis } & VEGF & $\begin{array}{l}\text { Vascular endothelial growth } \\
\text { factor }\end{array}$ \\
\hline & NOS & Nitric oxide synthase \\
\hline & LEP & Leptin \\
\hline & $\angle R P 1$ & LDL-receptor-related protein 1 \\
\hline & $A D M$ & Adrenomedullin \\
\hline & $T G F-\beta 3$ & Transforming growth factor- $\beta 3$ \\
\hline Erythropoiesis & $E P O$ & Erythropoietin \\
\hline \multirow{7}{*}{$\begin{array}{l}\text { Anaerobic glycolytic } \\
\text { metabolism }\end{array}$} & GLUT1/3 & Glucose transporter 1 and 3 \\
\hline & $H K 1 / 2$ & Hexokinase 1 and 2 \\
\hline & PFK & 6-phosphofructo-2-kinase \\
\hline & FBPase & Fructose-2,6-bisphosphatase \\
\hline & PKM & $\begin{array}{l}\text { Pyruvate kinase muscle isozymes } \\
\text { (M1/M2) }\end{array}$ \\
\hline & PDK1 & Pyruvate dehydrogenase kinase 1 \\
\hline & LDHA & Lactate dehydrogenase A \\
\hline \multirow[t]{2}{*}{ pH regulation } & MCT4 & Monocarboxylate transporter 4 \\
\hline & CA9 & $\begin{array}{l}\text { Membrane-associated carbonic } \\
\text { anhydrase IX }\end{array}$ \\
\hline \multirow[t]{8}{*}{$\begin{array}{l}\text { Cell proliferation and } \\
\text { survival }\end{array}$} & C-MYC & $\begin{array}{l}\text { Myelocytomatosis virus } \\
\text { oncogene cellular homolog }\end{array}$ \\
\hline & IGF2 & Insulin-like growth factor 2 \\
\hline & IGF-BP 1/2/3 & IGF-binding protein $1 / 2 / 3$ \\
\hline & ID2 & DNA-binding protein inhibitor \\
\hline & $A D M$ & Adrenomedullin \\
\hline & iNOS & Inducible nitric oxide synthase \\
\hline & $T G F-a$ & Transforming growth factor a \\
\hline & VEGF & $\begin{array}{l}\text { Vascular endothelial growth } \\
\text { factor }\end{array}$ \\
\hline \multirow{3}{*}{$\begin{array}{l}\text { Inflammation and } \\
\text { immunity }\end{array}$} & $T N F-a$ & Tumor necrosis factor a \\
\hline & Rags & $\begin{array}{l}\text { Recombination activating gene } \\
\text { products }\end{array}$ \\
\hline & TASK-2 & Potassium channels in B cells \\
\hline \multirow[t]{3}{*}{ Cancer metastasis } & FN1 & Fibronectin 1 \\
\hline & LOXL2 & Lysyl oxidase-like 2 \\
\hline & UPAR & $\begin{array}{l}\text { Urokinase plasminogen activator } \\
\text { receptor }\end{array}$ \\
\hline
\end{tabular}

Although general protein translation decreases during hypoxia, HIF- $1 \alpha$ continues to be translated. This event has been confirmed by several studies using translation and proteasomal inhibitors, and reporter assays with the 5 ' UTR of HIF1A gene. However, the exact mechanism by which $H I F 1 A$ is selectively translated during hypoxia remains unclear. One postulated mechanism includes the internal ribosome-entry-site (IRES) element within the 5' UTR of the HIF- $1 \alpha$ mRNA $^{41}$. The IRES element forms special secondary or tertiary structures, enabling ribosome binding in the absence of the eIF4F cap-binding complex. RNA-binding proteins, such as polypyrimidine tract-binding protein (PTB) and HuR, also bind the 3' UTR and 5' UTR of the HIF- $1 \alpha$ mRNA, respectively, promoting HIF- $1 \alpha$ translation ${ }^{42}$. HIF- $1 \alpha$ protein synthesis is also regulated indirectly by growth factor-induced signal transduction (Fig. 1a), including the phosphatidylinositol 3 kinase (PI3K), mitogen-activated protein kinase (MAPK), and calcium signaling pathways ${ }^{25}$. However, the effects of translational modulators are still controversial and remain to be clarified.

HIF- $1 \alpha$ activity is regulated by proteasomal degradation induced by specific post-translational modifications: hydroxylation, acetylation, SUMOylation, and phosphorylation $^{24,25}$. Under normoxic conditions, the von HippelLindau tumor suppressor (pVHL)-mediated ubiquitin proteasome pathway rapidly degrades HIF- $1 \alpha$; however, under hypoxic conditions, HIF- $1 \alpha$ protein degradation is prevented and the activated $\alpha$ subunit forms a heterodimer with the $\beta$ subunit to function as a transcription factor (Fig. 1c). The phosphorylation of a coactivator, p300/CBP, by ERK1/2 promotes HIF- $1 \alpha$-dependent transactivation of target genes (Fig. 1b). The oxygen sensitivity of the HIF pathway is conferred by prolyl hydroxylase (EGLN 1-3, also known as PHD 1-3) and HIF prolyl hydroxylase $(\mathrm{HPH})^{25,43}$. These hydroxylases are involved in the hydroxylation of HIF-1 $\alpha$ proline residues (Pro402 and Pro564) using oxygen as a substrate (Fig. 1d). In addition to oxygen, this reaction requires 2oxoglutarate as a cosubstrate and ascorbate and iron as cofactors $^{22}$. Because PHDs and HPH use iron as a cofactor, iron chelators, such as desferrioxamine (DFO), effectively inhibit PHDs and stabilize HIF- $1 \alpha^{44}$. Similarly, cobalt chloride $\left(\mathrm{CoCl}_{2}\right)$ is an well-known hypoxia mimetic agent that blocks the catalytic activity of PHDs by competing with the bivalent ion ${ }^{45}$, leading to HIF- $1 \alpha$ stabilization ${ }^{46,47}$.

The pVHL protein associates with elongins $\mathrm{B}$ and $\mathrm{C}$, cullin 2, and ring-box 1 (Rbx1) to form an E3 ubiquitinligase complex, pVHL-Elongin B/C-Cullin 2 (VEC), and subsequently ubiquinates the $\alpha$ subunit of HIF-1. The pVHL protein functions as the substrate-docking interface that specifically recognizes prolyl-hydroxylated HIF$1 \alpha^{48,49}$. Ubiquitin-tagged HIF- $\alpha$ proteins are subsequently 
Table 2 Factors that regulate the abundance and activity of HIF-1a

\begin{tabular}{|c|c|c|}
\hline Regulation stage & Modulators & Change in the level of HIF-1a \\
\hline \multirow[t]{4}{*}{ Transcription } & NFKB & $\uparrow$ \\
\hline & STAT3 & $\uparrow$ \\
\hline & p300/CBP & $\uparrow$ \\
\hline & ROS (by activating NFKB) & $\uparrow$ \\
\hline \multirow[t]{5}{*}{ Translation } & IRES elements & $\uparrow$ \\
\hline & RNA-binding proteins (PTB and HuR) & $\uparrow$ \\
\hline & PI3K and MAPK signaling pathways & $\uparrow$ \\
\hline & Calcium signaling & $\uparrow$ \\
\hline & miRNAs & $\uparrow$ \\
\hline \multirow[t]{11}{*}{ Post-translational degradation } & PHD, pVHL, OS-9 and SSAT2 (oxygen-dependent) & $\downarrow$ \\
\hline & ARD-1 (arrest-defective-1: oxygen-dependent acetylation of HIF-1a) & $\downarrow$ \\
\hline & Mdm2-p53 (ubiquitination and proteasomal degradation, oxygen-independent) & $\downarrow$ \\
\hline & EPF UCP (degrades pVHL, oxygen-dependent) & $\uparrow$ \\
\hline & VDU2 (de-ubiquitinates HIF-1a, oxygen-dependent) & $\uparrow$ \\
\hline & SUMOylation by RSUME (oxygen-dependent) & $\uparrow$ \\
\hline & DeSUMOylation by SENP1 (oxygen-dependent) & $\uparrow$ \\
\hline & Calcineurin A ( $\mathrm{Ca}^{2+}$-dependent dephosphorylation of RACK1, oxygen-independent) & $\uparrow$ \\
\hline & Hsp90 (oxygen-independent) & $\uparrow$ \\
\hline & RACK1 and SSAT1 (oxygen-independent) & $\downarrow$ \\
\hline & GSK3 $\beta$ and FoxO4 (regulated by PI3K and PTEN, oxygen-independent) & $\downarrow$ \\
\hline
\end{tabular}

* $\uparrow$ indicates an increase, $\downarrow$ indicates a decrease, and $\uparrow$ indicates either an increase or decrease in HIF- 1 a levels

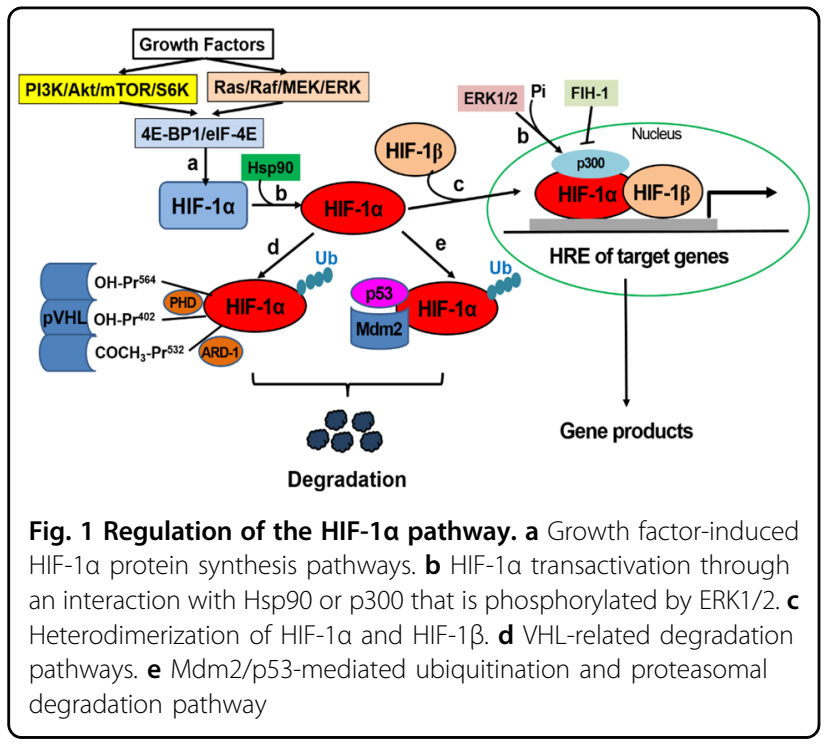

degraded by the common $26 \mathrm{~S}$ proteasome (Fig. 1d) 23,50 . This hydroxylation only occurs under normoxic conditions, i.e., in the presence of oxygen. Therefore, during hypoxia, HIF- $1 \alpha$ escapes degradation and dimerizes with the common and constitutively stable HIF- $1 \beta$ protein (Fig. 1c). Acetylation of HIF-1 $\alpha$ at Lys532 by arrestdefective-1 (ARD-1) also facilitates its recognition by pVHL and subsequent degradation under normoxic conditions (Fig. 1d) ${ }^{51}$. Other regulators of HIF-1 $\alpha$ include osteosarcoma-9 (OS-9) and spermidine/spermine- $\mathrm{N}^{1}$ acetyltransferase 2 (SSAT2). OS-9 forms a ternary complex with PHDs and HIF- $1 \alpha$, promoting proline hydroxylation and subsequent pVHL-dependent HIF- $1 \alpha$ degradation. SSAT2 binds to pVHL and elongin C, thereby stimulating HIF-1 $\alpha$ ubiquitination and degradation. On the other hand, Mdm2/p53-mediated ubiquitination and proteasomal degradation pathways are involved in regulating HIF- $1 \alpha$ levels in an oxygenindependent manner (Fig. 1e).

HIF- $1 \alpha$ activity is controlled via the degradation of pVHL by a member of the E2 enzyme family, endemic pemphigus foliaceus ubiquitin carrier protein (E2-EPF $\mathrm{UCP}$ ), and de-ubiquitination of HIF- $1 \alpha$ by the pVHLinteracting de-ubiquitylating enzyme (VDU2, USP20). 
However, the role of hypoxia-induced SUMOylation of HIF-1 $\alpha$ remains unclear ${ }^{25}$. In general, hypoxia results in HIF-1 $\alpha$ SUMOylation, which facilitates the recognition of HIF- $1 \alpha$ by the pVHL E3 ligase complex, leading to HIF- $1 \alpha$ degradation. Sentrin specific peptidase 1 (SENP1), a nuclear SUMO protease, reverses this modification, resulting in its stabilization during hypoxia ${ }^{52}$. However, the RWD-containing SUMOylation enhancer (RSUME)induced SUMOylation of HIF- $1 \alpha$ results in increased HIF- $1 \alpha$ protein levels and transactivation ${ }^{53}$.

The stability of the HIF- $1 \alpha$ protein is also regulated by oxygen-independent modulators, including a calciumdependent and calmodulin-dependent serine/threonine protein phosphatase (calcineurin A), a heat-shock protein (Hsp90), receptor of activated protein kinase C (RACK1) and GSK3 $\beta$. Calcineurin A dephosphorylates RACK1, thus inhibiting RACK1 dimerization and RACK1dependent HIF- $1 \alpha$ degradation ${ }^{54}$. Hsp90 binds to HIF$1 \alpha$, stabilizing the protein. Hence, Hsp90 inhibitors, such as 17-(allylamino)-17-demethoxygeldanamycin (17AAG), cause oxygen-independent HIF- $1 \alpha$ degradation. In contrast, the RACK1 homodimer binds to HIF- $1 \alpha$ upon Hsp90 inhibition (by 17AAG) and recruits components of the E3 ligase complex. SSAT1 (inactive) stabilizes the RACK1-HIF- $1 \alpha$ interaction, leading to HIF- $1 \alpha$ ubiquitination and degradation ${ }^{55}$. GSK3 $\beta$ phosphorylates HIF- $1 \alpha$, leading to its ubiquitination and degradation. Hypoxia might inhibit the PI3K/Akt pathway, causing the activation of GSK3 $\beta$ and FoxO4 to subsequently decrease HIF$1 \alpha$ levels $^{56}$. The PI3K pathway is activated by growth factors and inhibited by phosphatase and tensin homolog (PTEN) or serum depletion.

\section{Hypoxia and cancer}

Hypoxia plays important roles in cancer initiation, progression, and metastasis. Hypoxia is mainly observed in the tumor environment as the blood supply soon becomes inadequate for the rapidly growing tumor cells. Hypoxia activates HIF-1 to promote carcinogenesis and tumor growth by regulating the expression of genes involved in angiogenesis, glycolytic metabolism, and other biological mechanisms (Table 1). Once hydroxylated under normoxic conditions, HIF-1 binds to the tumor suppressor protein, $\mathrm{pVHL}$, which induces its proteasomal degradation. However, during hypoxia, the HIF-1 heterodimer undergoes stabilization and nuclear translocation, leading to the activation of several oncogenes. Hypoxic regulation of oncogenes by pVHL provides insights into cancer pathogenesis ${ }^{57}$. The role of $\mathrm{pVHL}$ in regulating HIF- $1 \alpha$ activity is evident in von Hippel Lindau disease. Inactivation of the $V H L$ gene induces the development of highly vascularized tumors in various organs, such as the kidney and central nervous system ${ }^{58}$. Recent evidence also support the hypothesis that hypoxia and hypoxia-related pathways play important roles in the development and progression of a subtype of renal cell carcinomas (clear cell RCC: 70-75\%), which is associated with the loss of pVHL function ${ }^{59}$, high levels of HIF-1, and thus deregulation of hypoxia pathways ${ }^{60-63}$. Interestingly, the two HIF- $\alpha$ proteins (HIF- $1 \alpha$ and HIF-2 $\alpha$ ) exert opposite effects on clear cell RCC, with HIF-1 $\alpha$ functioning as a tumor suppressor and HIF- $2 \alpha$ acting as an oncogene ${ }^{64}$. Moreover, because UCP ubiquitinates pVHL, leading to its degradation ${ }^{65}$, UCP overexpression causes pVHL degradation, resulting in HIF- $1 \alpha$ accumulation and increased tumor growth and metastasis. Conversely, UCP knockdown increases PVHL levels, causing a decrease in HIF-1 $\alpha$ levels and inhibiting tumor growth ${ }^{65}$. In addition to the pVHL, HIF- $1 \alpha$ and UCP proteins, a variety of modulators of HIF-1 $\alpha$ (Table 2 ) have been implicated in carcinogenesis and cancer metastasis, as recently reviewed by Aldo and Elisabetta ${ }^{66}$.

\section{Differences in the cellular responses to sustained hypoxia and intermittent hypoxia}

Interestingly, the inhibition of mitochondrial function reverses the hypoxia-induced HIF pathway. These observations prompted researchers to propose a role for the mitochondria in cellular oxygen sensing. Two models have been proposed to describe the role of mitochondria in oxygen sensing, implicating ROS and oxygen redistribution, respectively ${ }^{1}$. These two models may complement each other and facilitate the rapid and dynamic activation of the HIF pathway in response to hypoxia.

Under normoxic conditions, mitochondrial respiration consumes greater than $90 \%$ of the oxygen in humans. The remaining oxygen $(\sim 10 \%)$ is utilized for HIF- $1 \alpha$ degradation. In response to sustained hypoxia, the mitochondria consume almost all the oxygen and remove free cytosolic oxygen, causing the rapid stabilization of HIF$1 \alpha$. Activated HIF- $1 \alpha$ leads to the increased transcription of a number of genes, such as VEGF, EPO, and inducible nitric oxide synthase (iNOS). These factors participate in the adaptive response to hypoxia by increasing tissue perfusion and oxygenation, and hence aid in the recovery from the initial hypoxic insults. However, the molecular responses activated by intermittent hypoxia differ from those activated by sustained hypoxia. In intermittent hypoxia, a free oxygen deficit is not sufficiently established to allow HIF-1 $\alpha$ stabilization; instead, intermittent hypoxia results in the activation of $\mathrm{NF \kappa B}$, possibly through mitochondrial stress, triggering the production of inflammatory mediators, such as TNF- $\alpha$ (Fig. 2$)^{67}$. The level of HIF- $1 \alpha$ is also regulated transcriptionally in an NFKB-dependent manner ${ }^{36}$. Intermittent hypoxia may cause a delayed increase in HIF- $1 \alpha$, resulting in the activation of NFKB-driven inflammation, possibly as a result of oxidative stress ${ }^{67-70}$. 


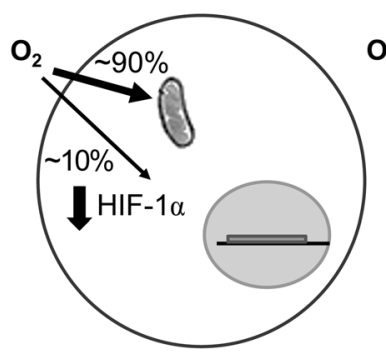

Normoxic conditions

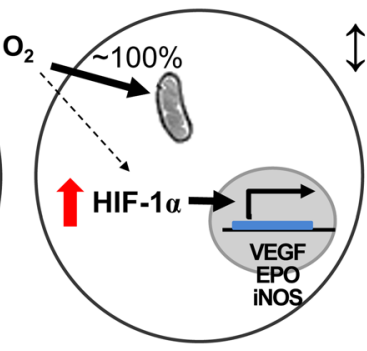

Sustained hypoxia

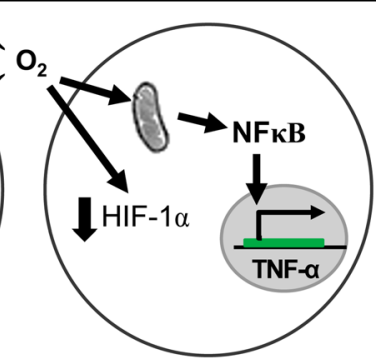

Intermittent hypoxia

Fig. 2 Hypoxia and HIF-1a stabilization. Under normoxic conditions, mitochondrial respiration consumes more than 90\% of oxygen in humans. The remaining cytosolic oxygen (approximately 10\%) is sufficient for HIF-1a degradation. During sustained hypoxia, the mitochondria use up almost all the oxygen and the free cytosolic oxygen is exhausted, causing HIF-1a stabilization. Activated HIF-1a induces the transcription of many genes, such as VEGF, EPO, and iNOS. However, the response to intermittent hypoxia differs from the response to sustained hypoxia. Intermittent hypoxia may not remove a sufficient amount of cytosolic free oxygen to allow HIF-1a stabilization; instead, intermittent hypoxia activates NFKB probably through mitochondrial stress, resulting in the production of TNF-a, an inflammatory mediator

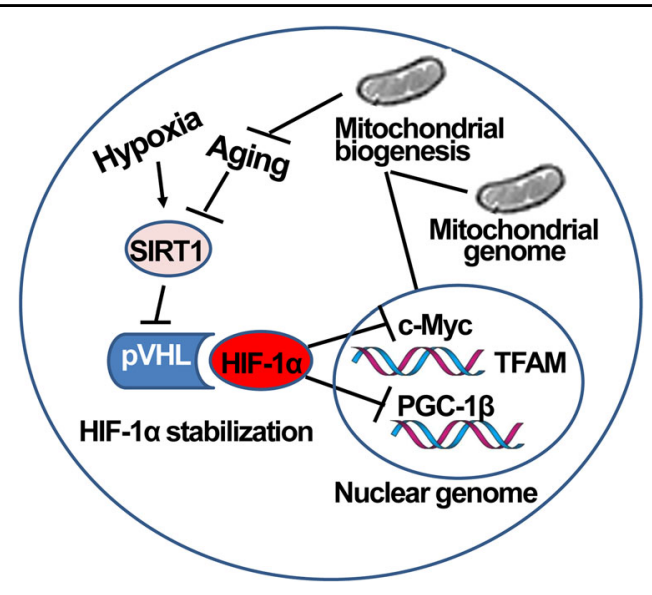

Fig. 3 Regulation of mitochondrial biogenesis and aging by SIRT1 and HIF-1a. During the aging process, the activity of SIRT1 in the nucleus is reduced, which decreases pVHL levels and subsequently stabilizes HIF-1a. Activated HIF-1a reduces c-Myc activity and subsequently reduces the transcription of TFAM, which is required for replication, transcription, and maintenance of mitochondrial biogenesis. PGC-1 $\beta$ activity is also inhibited upon its interaction with HIF-1a, resulting in the downregulation of mitochondrial genes

\section{HIF-1a and aging}

Cross-talk between the HIF-1a and SIRT1 pathways during aging

Aging is a degenerative process leading to cellular dysfunction, tissue failure, and a deterioration of bodily functions. Two well-known theories, the "free radical theory of aging" and "mitochondrial theory of aging" (Bereiter-Hahn, 2014), claim that mitochondria-induced oxidative damage results in detrimental effects on mitochondrial function, leading to ROS production and inflicting further injury ${ }^{71,72}$.

During the aging process, HIF- $1 \alpha$ induces a deficit in mitochondrial biogenesis (Fig. 3), which impairs energy- dependent cellular processes, including cell and tissue repair $^{73}$. The subsequent accumulation of ROS, oxidation of lipids and proteins, and mutations in mitochondrial DNA accelerate the aging process by inducing a deterioration of cellular energetics, the cellular redox state, calcium homeostasis, and cell signaling ${ }^{73}$. Therefore, the mechanisms regulating HIF-1 $\alpha$ have been implicated in the prevention of premature cellular senescence and the pathogenesis of many aging-related chronic diseases.

SIRT1 is a member of the sirtuin family, sharing homology with Saccharomyces cerevisiae silent information regulator 2 (Sir2), a highly conserved protein with dual enzymatic activities: nicotinamide adenine dinucleotide $\left(\mathrm{NAD}^{+}\right)$-dependent deacetylases and ADPribosyltransferase activities. Although this family is composed of seven members, SIRT1 $-7^{74}$, SIRT1 is the most extensively studied member due to its involvement in the mechanisms regulating various important biological and pathological processes, including apoptosis, inflammation, and senescence ${ }^{75}$, which are associated with aging-related diseases. Studies of the transcriptional regulation of the SIRT1 gene in metabolism have revealed critical roles for peroxisome proliferator-activated receptor (PPAR)- $\beta / \delta$ and the canonical Sp1 binding site in the 5' SIRT1 promotor $^{76}$. Sp1 overexpression significantly enhances SIRT1 promoter activity by binding to the Sp1 element, as confirmed by EMSA and ChIP assays and experiments using a specific Sp1 antagonist, mithramycin. Two miRNAs, miR-34a and miR-93, are known to be upregulated; however, the expression of their candidate targets, Sp1 and SIRT1, are reduced during aging ${ }^{77}$. A reduction in Sp1 activity might be responsible for the decrease in SIRT1 transcription, and subsequent suppression of angiotensin II-induced cardiac hypertrophy by flavonol (-)-epicatechin ${ }^{78}$. 
Interestingly, the level of SIRT1 decreases in both transcriptional and posttranscriptional stages during aging, which attenuates mitochondrial biogenesis and causes aging-related diseases. Aging-associated reductions in the nuclear energy state and $\mathrm{NAD}^{+}$levels decrease SIRT1 activity, resulting in diminished pVHL levels and the stabilization of HIF-1 $\alpha$ (Fig. 3). Conversely, overexpression of SIRT1 promotes mitochondrial biogenesis by deacetylation, resulting in the activation of HIF- $1 \alpha^{79}$ (Fig. 3) and PPAR $\gamma$ co-activator $1 \alpha(\mathrm{PGC}-1 \alpha)^{80}$. The upregulation of SIRT1 has been implicated in the prevention of premature cellular senescence and the pathogenesis of many aging-related chronic diseases ${ }^{81-83}$. SIRT1 is the factor that is activated by caloric restriction and natural polyphenolic compounds, such as resveratrol, which is responsible for increasing longevity. Therefore, overexpression of SIRT1 results in delayed aging phenotypes and an extension of the lifespan, whereas inhibition of SIRT1 abrogates the extension of the lifespan in mice ${ }^{84}$.

HIF- $1 \alpha$ regulates mitochondrial biogenesis, and modulation of the nuclear-mitochondrial communication during aging depends on PGC- $1 \alpha^{79}$. HIF- $1 \alpha$ accumulates in the nucleus of both adult SIRT1 knockout mice and SIRT1-silenced primary myoblasts ${ }^{85}$. The increased HIF$1 \alpha$ levels observed during aging or in response to SIRT1 knockout activated the Mxil gene, which encodes a cMyc transcriptional repressor. The Mxil gene product restricts the interaction between $\mathrm{c}-\mathrm{Myc}$ and mitochondrial transcription factor A (TFAM) that is critical for replication, transcription, and maintenance of mitochondrial biogenesis ${ }^{86}$, further decreasing the TFAM promoter activity and suppressing mitochondrial biogenesis ${ }^{79}$.

SIRT1 mRNA transcription is also coupled with hypoxia during the aging process (Fig. 3). Feedback control mechanisms regulating levels of the SIRT1 transcript include the p53/forkhead box O-3a (FoxO3a)-SIRT1 pathway, FoxO1-SIRT1 pathway and HIF-1 $\alpha / \mathrm{HIF}-2 \alpha-$ SIRT1 pathway ${ }^{73}$. During acute hypoxia, HIF-1 $\alpha$ and HIF$2 \alpha$ accumulate stably, interact with Hsp90, and directly bind to HREs in the SIRT1 promoter. During aging, PPAR1 activity is substantially enhanced, leading to $\mathrm{NAD}^{+}$depletion and thereby inhibiting SIRT1 activity. In addition, the hypermethylated in cancer 1 protein (HIC1) and C-terminal-binding protein 1 (CtBP) complex associates with SIRT1 to repress SIRT1 transcription. A repressor complex, containing PPAR $\gamma$ or PPAR $\beta$ and SIRT1, binds to specific sites in the SIRT1 promoter and directly suppresses transcription induced by exogenous stressors. The SIRT1 promoter region consists of a FoxO1 core binding repeat motif, and FoxO1-mediated SIRT1 transcription results in an increased expression of SIRT1. In response to DNA damage, acetylated E2F1 enhances the binding of FoxO1 to the SIRT1 promoter region, increasing SIRT1 transcription.

\section{Cross-talk between the SIRT1 and AMPK pathways during hypoxia and aging}

AMPK is a heterotrimeric serine/threonine protein kinase composed of a catalytic $\alpha$ subunit and regulatory $\beta$ and $\gamma$ subunits. AMP binds to the $\gamma$ subunit and activates AMPK by promoting Thr172 phosphorylation of the $\alpha$ subunit mediated by liver kinase B1 (LKB1) ${ }^{87}$. When the $\alpha$ subunit is phosphorylated on Ser485 by other upstream kinases, such as insulin-activated Akt, cAMP-dependent protein kinase (PKA), and RAS/MEK/ERK pathways, it is rendered inactive ${ }^{88}$. AMPK is activated by other serine/ threonine kinases, such as $\mathrm{Ca}^{2+} /$ calmodulin-dependent protein kinase $\beta$ and transforming growth factor- $\beta$ activated kinase 1 , and inhibited by several protein phosphatases, such as PP1, PP2A, and PP2C ${ }^{89}$.

AMPK is an important regulator of energy metabolism, stress resistance, and cellular proteostasis. AMPK also links energetics to longevity ${ }^{90}$. According to the results from several studies, the activation capacity of AMPK signaling decreases with aging, which impairs the maintenance of efficient cellular homeostasis and accelerates the aging process ${ }^{89}$. However, aging also enhances AMPK activation under certain conditions, suggesting that the aging process affects AMPK activation in a contextdependent manner.

AMPK activation extends the lifespan by reducing oxidative stress through an increase in thioredoxin levels, by reducing endoplasmic reticulum stress and inflammatory disorders, and by promoting autophagic clearance during the aging process ${ }^{91}$. In addition, active AMPK is involved in several aging-associated processes, such as cancer, insulin resistance, and osteoarthritis, characterized by defects in mitochondrial biogenesis ${ }^{92}$. Similar to SIRT1, activated AMPK modulates mitochondrial biogenesis via PGC- $1 \alpha$. Mitochondrial biogenesis is compromised in humans with osteoarthritis and aging chondrocytes, which is reversed by the activation of the AMPK-SIRT1PGC1 $\alpha$ pathway ${ }^{92}$.

Hypoxia is known to activate AMPK directly by increasing the AMP:ATP ratio or indirectly via SIRT1 activation (Fig. 4). LKB1 is activated by SIRT1 and promotes the phosphorylation and activation of the catalytic $\alpha$-subunit of AMPK $^{93}$. Activated AMPK directly phosphorylates and activates $\mathrm{p} 53^{94}$, which induces the upregulation of cyclin-dependent protein kinase inhibitors and pro-apoptotic genes, such as $\mathrm{p} 21$ and Bax, respectively ${ }^{95}$, resulting in cell cycle arrest and apoptosis ${ }^{96}$. AMPK also induces the upregulation of $\mathrm{p} 21$ and Bax via the acetylation of $\mathrm{p} 53$ by AMPK-induced phosphorylation and inactivation of $\operatorname{SIRT}^{95}$. In skeletal muscle, exerciseactivated AMPK directly increases the phosphorylation of Thr177 and Ser538 and activity of PGC- $1 \alpha$ in the nucleus, which triggers the transcription of mitochondrial genes, such as nuclear respiratory factors (NRFs) and estrogen- 


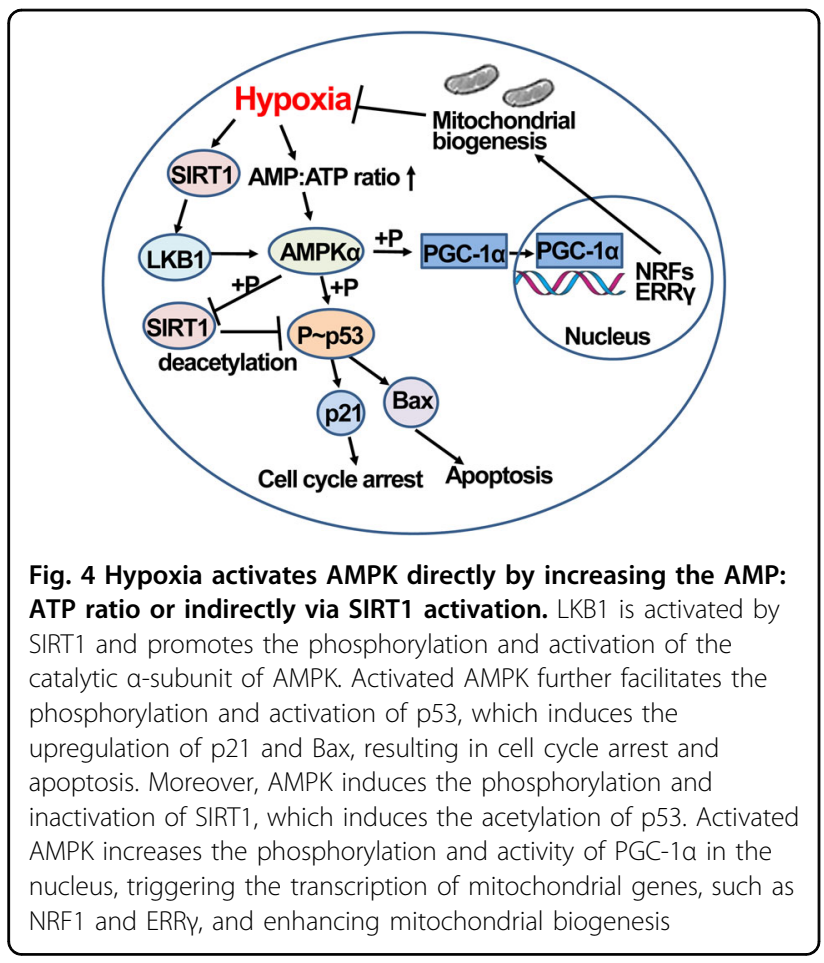

related receptor $\gamma(E R R \gamma)$, and strengthens mitochondrial biogenesis (Fig. 4) $)^{97}$. Meanwhile, activated AMPK increases the expression of PGC- $1 \alpha$ protein and subsequent formation of a complex with SIRT1-MyoD, which in turn binds to the promoter of PGC- $1 \alpha$, creating a positive feedback loop for PGC-1 $\alpha$ expression ${ }^{98}$. In addition to SIRT1-mediated deacetylation of PGC- $1 \alpha$, acetylation of PGC- $1 \alpha$ by the acetyltransferase general control nonderepressible 5 (GCN5) might be another mechanism controlling PGC- $1 \alpha$ activity. Acetylation of PGC-1 $\alpha$ decreases mitochondrial biogenesis ${ }^{99}$.

\section{Cross-talk between the AMPK and mTOR-ULK1 pathways during hypoxia and aging}

Furthermore, relationships among AMPK, mTOR, ULK1 (the key regulatory protein required to initiate mammalian autophagy as the mammalian homologs of ATG1), and autophagy (the process by which cellular components are self-degraded) have been suggested under hypoxic conditions ${ }^{100}$. mTOR is a serine/threonine protein kinase that influences organismal lifespan in various species, including mammals ${ }^{12,101}$. mTOR exists in two distinct protein complexes, mTORC1 and mTORC $2^{102}$. Rapamycin-sensitive mTORC1 regulates protein synthesis and cell growth through the phosphorylation of p70 ribosomal S6 kinase 1 (p70S6K1: Thr389) and initiation factor 4E-binding protein 1 (4E-BP1: Thr37/46) $)^{103,104}$. The PI3K/Akt pathway is one of classic upstream pathways of mTORC1 signaling, and the tuberous sclerosis protein 1 and 2 (TSC1/2) complex is a

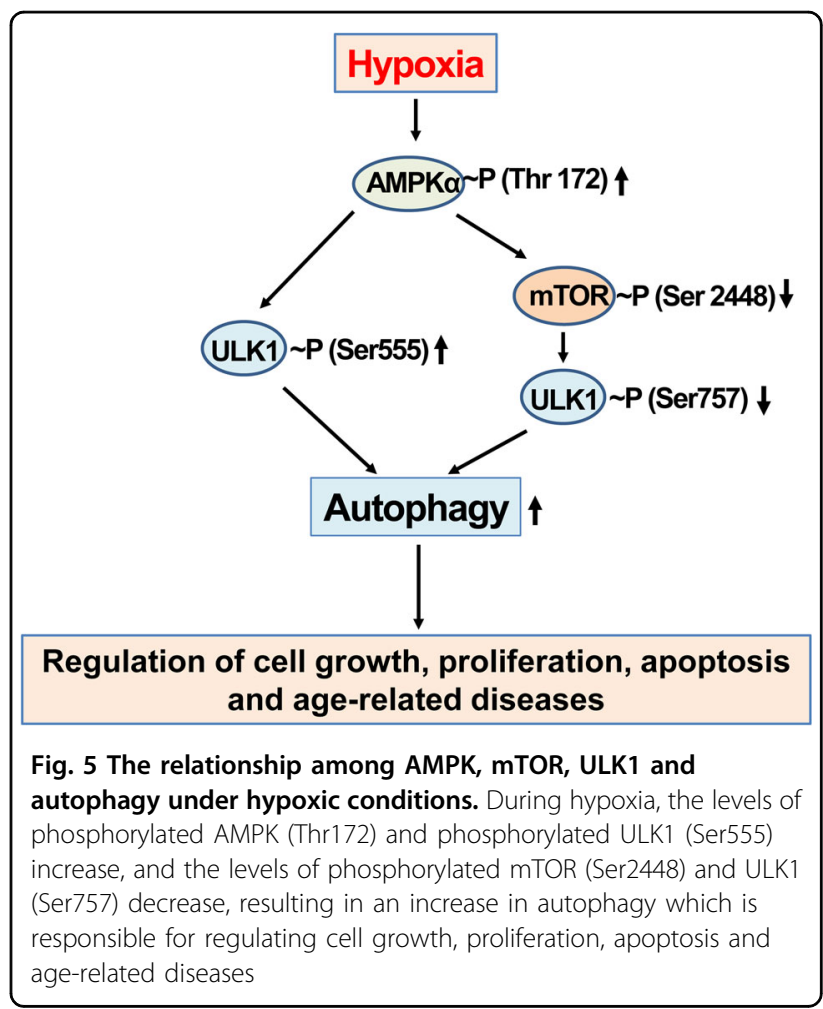

negative regulator of mTOR. Akt inactivates TSC2 via phosphorylation $^{105}$, whereas AMPK phosphorylates and activates $\mathrm{TSC} 2^{106}$.

Moreover, AMPK phosphorylates ULK1 either through direct or indirect pathways (Fig. 5). AMPK enhances autophagy by directly phosphorylating Ser555 in ULK $1^{107,108}$. Meanwhile, AMPK inhibits the activity of mTOR to regulate cell growth and proliferation ${ }^{106,109-111}$ and mTOR inhibits autophagy by phosphorylating ULK1 at Ser757 707,112 . During hypoxia, the levels of mTOR, phosphorylated mTOR (Ser2448), and phosphorylated ULK1 (Ser757) are decreased, promoting autophagy, which is responsible for regulating cell growth, proliferation, apoptosis, and age-related diseases. Autophagy is initially increased as a compensatory response to hypoxia. Further enhancements in autophagy by chemical modulators may decrease cell growth and proliferation, increase autophagic cell death and apoptosis, and consequently regulate age-related diseases ${ }^{100}$.

Both the HIF and AMPK signaling pathways are evolutionarily conserved and play major roles in enabling survival during severe stress conditions, such as hypoxia and energy deficiency. The HIF-1-induced and AMPKinduced survival network includes protein kinases and other factors, such as SIRT1, mTOR, and ULK1, as well as several transcription factors, such as p53, FoxO, NFкB, and NRF2. HIF1 and AMPK activation increase not only the healthy lifespan but also the progression of 
tumorigenesis in a context-dependent manner ${ }^{113}$. HIF- $1 \alpha$ increases angiogenesis by promoting the Src-dependent expression of VEGF in pancreatic and prostate carcinomas to help the cells adapt to harsh conditions ${ }^{114}$. Src activation leads to increased steady-state levels of HIF- $1 \alpha$ and increased phosphorylation of STAT3. Both STAT3 and HIF- $1 \alpha$ bind to the promoter of the VEGF gene, where they form a complex with the transcriptional coactivators, including $\mathrm{p} 300 / \mathrm{CBP}$ and Ref-1/APE.

\section{Cross-talk between the AMPK and NFKB pathways during hypoxia and aging}

Although the AMPK signaling pathway functions to improve energy metabolism and autophagy, it inhibits chronic stress-linked inflammation ${ }^{115}$. AMPK also inhibits endoplasmic reticulum and oxidative stress, which are involved in metabolic disorders and in the aging process. A low-level of chronic inflammation is observed during the aging process and the AMPK signaling pathway inhibits NFkB-induced inflammatory responses (Fig. 6) ${ }^{115}$. $\mathrm{NF} \kappa \mathrm{B}$ signaling is inhibited by the AMPK-dependent phosphorylation of several downstream targets, such as

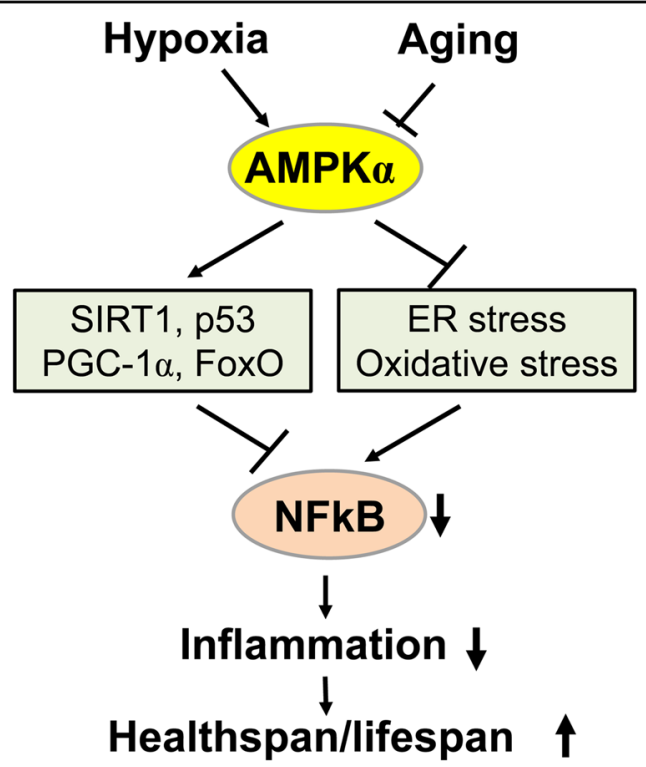

Fig. 6 The AMPK pathway links hypoxia and aging to NFKB signaling and inflammation. Hypoxia activates AMPK. In contrast, aging appears to inhibit the expression and activity of AMPKa. Activated AMPK in turn stimulates SIRT1, p53, PGC-1a and FoxO, which inhibit NFKB signaling via different mechanisms. AMPK also inhibits the induction of ER and oxidative stress, which are responsible for activating NFKB signaling. NFKB is inhibited by the activated AMPK pathway, resulting in a reduction of inflammatory responses, which affect the healthspan and lifespan. ${ }^{a}$ Medizinische Klinik 4 and Translational Research Center, Universitätsklinikum Erlangen und Friedrich-Alexander-Universität (FAU) Erlangen-Nürnberg, Erlangen, Germany
SIRT1, p53, PGC1 $\alpha$, and FoxO. AMPK-induced suppression of inflammatory responses has been found to augment the healthspan and lifespan.

The role of AMPK in cancer suppression has also been reviewed extensively ${ }^{116}$. Similar to aging, cancer also involves the induction of inflammation, and thus AMPK may play a role in preventing inflammatory responses in cancer cells. Therefore, the LKB1-AMPK signaling pathway was shown to be a powerful tumor suppressor pathway. AMPK agonists, such as metformin, hold promise as potent cancer drugs ${ }^{116,117}$. In agreement with this hypothesis, metformin inhibits the mTORC1 pathway via AMPK-dependent and AMPK-independent pathways, thereby inhibiting cancer cell growth and development ${ }^{113,118}$.

\section{Prenatal hypoxia and aging}

Recently, experimental, epidemiological, and clinical studies have suggested critical roles for gestational (prenatal) factors in brain development and functions in the postnatal period. The prenatal factors increase the risks of various CNS disorders, such as autism, schizophrenia, Down syndrome, epilepsy, and depression, and predispose aging individuals to the development of diabetes and neurodegenerative disorders, including Parkinson's and Alzheimer's diseases ${ }^{119}$. Prenatal factors affecting fetal brain development include tobacco and alcohol, poor nutrition, maternal stress, placental deficits, hormones, toxins, and infections ${ }^{120}$. Since pregnancy complications, abnormal pregnancy and labor often lead to an insufficient oxygen supply to the fetus, prenatal hypoxia during the critical periods of brain development is one of the

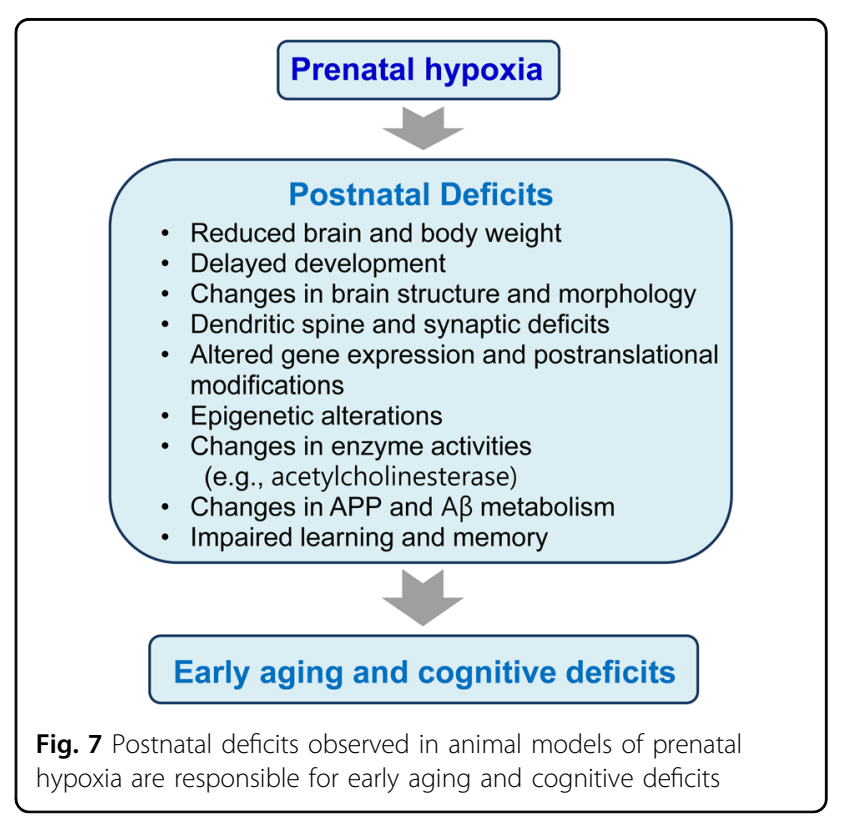


most important factors manifesting in early aging, mental retardation, and cognitive deficits at various postnatal stages of life ${ }^{120}$. An understanding of which type of changes are induced by prenatal hypoxia in the developing brain is important to prevent the development of CNS disorders later in life. These changes are outlined in Fig. 7.

In general, neuronal cells are more vulnerable to the effects of hypoxia than other types of cells ${ }^{121}$. Therefore, hypoxic insults to the brain have more significant consequences than in other organs and lead to severe pathologies ${ }^{122}$. Similarly, prenatal hypoxia may more severely affect the brain, resulting in a number of changes in its structural and functional properties ${ }^{123}$. Prenatal hypoxia reduces the action potentials and plasticity in the brain by preventing the formation of new contacts between cells and the propagation of neuronal signals, particularly in the cortex and hippocampus, which play important roles in learning and memory. Furthermore, prenatal hypoxia has a significant impact on the expression of a variety of genes, resulting in changes in the patterns of mRNA and protein expression and their posttranslational modifications, including protein misfolding and clearance. Among these proteins, acetylcholinesterase, a key enzyme of the cholinergic system, and amyloid precursor protein (APP) are significantly affected by prenatal hypoxia ${ }^{124,125}$. Because these proteins have important roles in brain function, hypoxia-induced disruption of their expression and metabolism can result in early cognitive dysfunctions and the subsequent development of neurodegeneration. A decrease in the activity of NEP and other amyloid- $\beta$ peptide $(A \beta)$-degrading enzymes was also observed after prenatal hypoxia, resulting in an $A \beta$ clearance deficit, accumulation of its toxic species, and subsequent neuronal cell death and neurodegeneration (Fig. 7).

In addition, prenatal hypoxia has been shown to impair the response of the developing organism to hypoxia in adulthood by inducing alterations in catecholaminergic components of the chemoafferent pathway contributing to impaired postnatal respiratory behavior ${ }^{126}$. Hypoxiainduced down-regulation of NEP might contribute to these alterations ${ }^{127}$. Adult rats exposed to intermittent hypoxia in the neonatal period exhibit an augmented carotid body and adrenal chromaffin cell response to hypoxia and irregular breathing, which are associated with increased oxidative stress ${ }^{128}$. The application of various approaches to restore expression of neuronal genes that are disrupted by prenatal hypoxia during postnatal development opens an avenue for the therapeutic compensation of cognitive dysfunctions and prevention of $A \beta$ accumulation in the aging brain and the model of prenatal hypoxia in rodents, which can be used as a reliable tool to assess their efficacy.

\section{OSA-induced intermittent hypoxia and aging}

OSA is a sleep-related breathing disorder characterized by repeated episodes of complete (apnea) or partial (hypoapnea) obstruction of the upper airway. OSA causes cyclical hypoxemia-reoxygenation and stimulates chemoreceptors, resulting in over-activation of SNA, bursts in systemic blood and arterial pulmonary pressures ${ }^{6}$, and concomitant cellular chronic intermittent hypoxia ${ }^{129}$. Long-term consequences of OSA or chronic intermittent hypoxia include molecular and cellular impairments that contribute to the accelerated progression and severity of many diseases, including cardiovascular diseases ${ }^{130,131}$, metabolic diseases ${ }^{132}$, neurological disorders ${ }^{131,133,134}$, cancer and aging ${ }^{135}$. The pathophysiological consequences and clinical manifestations of OSA or chronic intermittent hypoxia are summarized in Table 3.

Previously, hypoxia was shown to potentially contribute to cellular aging and the functional decline during the aging process. OSA-associated intermittent hypoxia may also accelerate cellular aging and age-related diseases by prompting the induction of nine hallmarks of aging: genomic instability, telomere shortening, epigenetic alterations, loss of proteostasis, deregulated nutrient sensing, mitochondrial dysfunction, cellular senescence, stem cell exhaustion, and altered cellular communication (Fig. 8) ${ }^{136}$.

Because aging is one of the risk factors for cancers and most hallmarks of aging have been shown to accelerate tumorigenesis, resulting in cancers, OSA is also postulated influence tumorigenesis and carcinogenesis ${ }^{135,137}$. Tumor physiology, animal and epidemiological human studies reveal a strong relationship between OSA and cancer. However, because current data relating OSA to neoplastic diseases remain scarce, more research on the impact of OSA on cancer-related aspects are needed.

\section{Summary}

Due to its crucial role in metabolism and survival, hypoxia has attracted the interest of many researchers. Hypoxia-inducible factors (HIFs) form an efficient and rapid oxygen sensing system, and effectively control the hypoxic responses, which induce the expression of several adaptive genes to increase the oxygen supply and support anaerobic ATP generation in eukaryotic cells. Agingassociated cumulative damages and degenerative changes lead to dysfunction and failure at the cellular and tissue levels, ultimately resulting in disorders of whole body function. Hypoxia potentially contributes to functional decline during the aging process. We must improve our understanding of the molecular and cellular responses to hypoxia to reduce the hypoxia-induced damage and senescence induction.

HIF pathways cross-talk with sirtuins, AMPK, and mTORC1-ULK1 pathways, which are involved in the 
Table 3 The pathophysiological consequences and clinical manifestations associated with obstructive sleep apnea or chronic intermittent hypoxia

1. Cardiovascular diseases

Pathophysiologic consequences

- Hypoxemia, cyclic hypercapnia and hypocapnia

- Nocturnal and diurnal hypertension

- Alterations in coronary blood flow

- Increased sympathetic activity

- Increased oxidative stress

- Increased inflammatory response (elevated levels of C-reactive protein,

NFKB, TNF-a, IL-8, IL-6, and EPO)

- Increased levels of adhesion molecules (ICAM-1)

- Endothelial dysfunction (increased big ET-1 or ET-1 level, reduced nitric

oxide level, and activation of the renin-angiotensin system)

- Increased coagulation (fibrinogen)

- Increased platelet aggregation

- Increased blood viscosity

2. Metabolic diseases

Pathophysiological consequences

- Activation of the sympathetic system

- Increased release of adipocyte-derived inflammatory mediators (IL-6, TNF-a, and leptin)

- Activation of hypothalamus-pituitary-adrenal axis (increased cortisol levels)

3. Neurological disorders

Pathophysiological consequences

- Increased activity of sympathetic neuron

- Increased oxidative stress

- Increased neuronal apoptosis within the cortex and CA1 region of the

hippocampus

- Increased expression of the COX-2 protein and gene

4. Cancer and aging

Pathophysiological consequences

- Increased oxidative stress

- Molecular and cellular impairments

- Accelerated cellular aging

5. Other functional defects

Pathophysiological consequences

- Reduced upper airway muscle endurance and impaired pharyngeal dilator - EMG responses to physiological stimulation

mechanisms regulating inflammation, mitochondrial biogenesis, cellular senescence, and organismal aging. The putative molecular mechanisms underlying the effects of hypoxia, including HIF- $1 \alpha$, AMPK, sirtuins, and mTORC1, are discussed in this manuscript. The levels of
Clinical manifestations

- Systemic hypertension

- Congestive heart failure (systolic and diastolic dysfunction)

- Coronary artery disease

- Arrhythmias

- Pulmonary hypertension

- Stroke

- Atherosclerosis

Clinical manifestations

- Glucose intolerance and insulin resistance

- Type 2 diabetes mellitus

- Metabolic syndrome

Clinical manifestations

- Depression

- Insomnia and/or excessive daytime somnolence

- Cognitive and neurobehavioral abnormalities: impairments in concentration, perception, memory, performance and learning

- Attention deficit hyperactive disorder (ADHD)

- Headaches

Clinical manifestations

- Tumors

- Cancer

Clinical manifestations

- Gastroesophageal reflux

- Genitourinary: nocturia and sexual dysfunction

- Toxemia (preeclampsia/eclampsia) during pregnancy

these proteins are controlled by both hypoxia and aging; the level of SIRT1 is decreased at both transcriptional and posttranscriptional levels during aging, which attenuates mitochondrial biogenesis and causes aging-related diseases. The activity of SIRT1 is also regulated by an aging- 


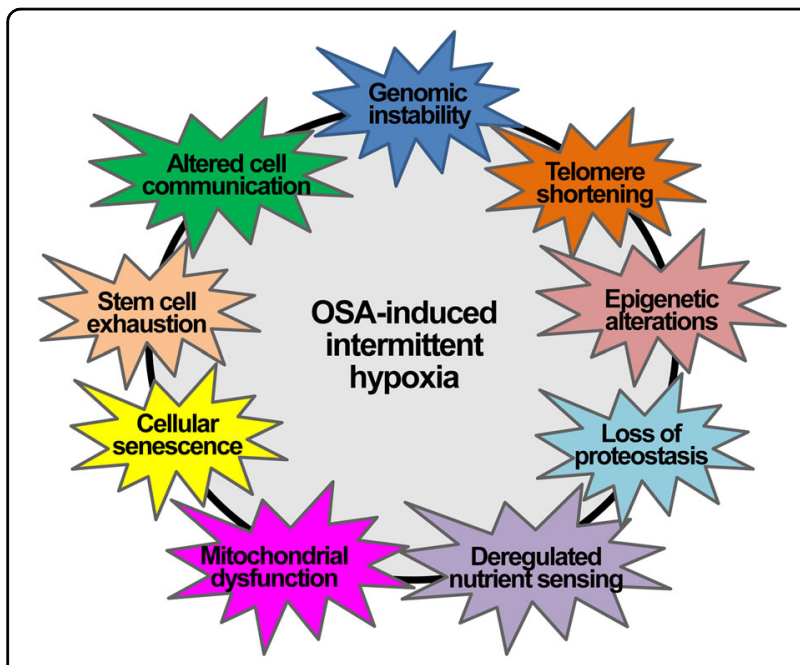

Fig. 8 The hypothetical impact of OSA-induced intermittent hypoxia on aging and aging-related diseases. OSA-associated intermittent hypoxia may promote the induction of the nine hallmarks of aging: genomic instability, telomere shortening, epigenetic alterations, loss of proteostasis, deregulated nutrient sensing, mitochondrial dysfunction, cellular senescence, stem cell exhaustion, and altered intercellular communication. These changes accelerate aging and aging-related diseases, such as cancer

associated reduction of the nuclear energy state and $\mathrm{NAD}^{+}$levels, resulting in diminished levels of the pVHL protein and stabilization of HIF-1 $\alpha$. Conversely, overexpression of SIRT1 promotes mitochondrial biogenesis and the subsequent activation of HIF- $1 \alpha$ and PGC- $1 \alpha$. The upregulation of SIRT1 may prevent premature cellular senescence and the pathogenesis of many agingrelated chronic diseases.

AMPK is an important regulator of energy metabolism, stress resistance, and cellular proteostasis. AMPK also links energetics to longevity and its activation extends the lifespan. The activation capacity of AMPK signaling decreases with aging, which impairs the maintenance of efficient cellular homeostasis and accelerates the aging process. Similar to SIRT1, activated AMPK modulates mitochondrial biogenesis via PGC- $1 \alpha$. A deficiency in mitochondrial biogenesis is reversed by the activation of the AMPK-SIRT1-PGC- $1 \alpha$ pathway. Hypoxia activates AMPK directly by increasing the AMP:ATP ratio or indirectly via SIRT1 activation. LKB1 is the protein activated by SIRT1, which promotes the phosphorylation and activation of the catalytic $\alpha$-subunit of AMPK.

Hypoxia contributes to cellular aging and the functional decline observed during the aging process. Prenatal hypoxia during the critical periods of brain development could manifest as early aging, mental retardation, and cognitive deficits in postnatal life. OSA-associated intermittent hypoxia may also accelerate cellular aging and aging-related diseases by prompting the induction of the nine hallmarks of aging: genomic instability, telomere shortening, epigenetic alterations, loss of proteostasis, deregulated nutrient sensing, mitochondrial dysfunction, cellular senescence, stem cell exhaustion, and altered cellular communication.

\section{Acknowledgements}

This work was supported by a grant from the Basic Science Research Program of the National Research Foundation of Korea (NRF) (No. 2017R1D1A1B03033499) funded by the Ministry of Education, Science and Technology of the Korean Government.

\section{Conflict of interest}

The author declares no conflict of interest.

\section{Publisher's note}

Springer Nature remains neutral with regard to jurisdictional claims in published maps and institutional affiliations.

Received: 6 December 2018 Accepted: 20 December 2018. Published online: 20 June 2019

\section{References}

1. Taylor, C. T. Mitochondria and cellular oxygen sensing in the HIF pathway. Biochem. J. 409, 19-26 (2008)

2. Sharp, F. R. \& Bernaudin, M. HIF1 and oxygen sensing in the brain. Nat. Rev. Neurosci. 5, 437-448 (2004).

3. Kemp, P. J. et al. Airway chemotransduction: from oxygen sensor to cellular effector. Am. J. Respir. Crit. Care Med. 166, S17-S24 (2002).

4. Kumar, G. K., Yu, R. K., Overholt, J. L. \& Prabhakar, N. R. Role of substance P in neutral endopeptidase modulation of hypoxic response of the carotid body. Adv. Exp. Med. Biol. 475, 705-713 (2000).

5. Caravagna, C. \& Seaborn, T. Oxygen sensing in early life. Lung 194, 715-722 (2016).

6. Jordan, A. S., McSharry, D. G. \& Malhotra, A. Adult obstructive sleep apnoea. Lancet 383, 736-747 (2014).

7. Semenza, G. L. Regulation of mammalian $\mathrm{O} 2$ homeostasis by hypoxiainducible factor 1. Annu. Rev. Cell Dev. Biol. 15, 551-578 (1999).

8. Semenza, G. L. HIF-1: mediator of physiological and pathophysiological responses to hypoxia. J. Appl. Physiol. 88, 1474-1480 (2000).

9. Antikainen, H., Driscoll, M., Haspel, G. \& Dobrowolski, R. TOR-mediated regulation of metabolism in aging. Aging Cell 16, 1219-1233 (2017).

10. Hong, S., Zhao, B., Lombard, D. B., Fingar, D. C. \& Inoki, K. Cross-talk between sirtuin and mammalian target of rapamycin complex 1 (mTORC1) signaling in the regulation of S6 kinase 1 (S6K1) phosphorylation. J. Biol. Chem. 289, 13132-13141 (2014).

11. Ruderman, N. B. et al. AMPK and SIRT1: a long-standing partnership? Am. J. Physiol. Endocrinol. Metab. 298, E751-E760 (2010).

12. Pan, H. \& Finkel, T. Key proteins and pathways that regulate lifespan. J. Biol. Chem. 292, 6452-6460 (2017).

13. Wang, G. L., Jiang, B. H., Rue, E. A. \& Semenza, G. L. Hypoxia-inducible factor 1 is a basic-helix-loop-helix-PAS heterodimer regulated by cellular $\mathrm{O} 2$ tension Proc. Natl Acad. Sci. USA 92, 5510-5514 (1995).

14. Jiang, B. H., Rue, E., Wang, G. L., Roe, R. \& Semenza, G. L. Dimerization, DNA binding, and transactivation properties of hypoxia-inducible factor 1. J. Biol. Chem. 271, 17771-17778 (1996).

15. lyer, N. V. et al. Cellular and developmental control of $\mathrm{O} 2$ homeostasis by hypoxia-inducible factor 1 alpha. Genes Dev. 12, 149-162 (1998).

16. Iyer, N. V., Leung, S. W. \& Semenza, G. L. The human hypoxia-inducible factor 1alpha gene: HIF1A structure and evolutionary conservation. Genomics $\mathbf{5 2}$ 159-165 (1998).

17. Semenza, G. L. Targeting HIF-1 for cancer therapy. Nat. Rev. Cancer 3, 721-732 (2003).

18. Jiang, B. H., Zheng, J. Z., Leung, S. W., Roe, R. \& Semenza, G. L. Transactivation and inhibitory domains of hypoxia-inducible factor 1alpha. Modul. Transcr. Act. Oxyg. Tens. J. Biol. Chem. 272, 19253-19260 (1997). 
19. Bruick, R. K. \& McKnight, S. L. A conserved family of prolyl-4-hydroxylases that modify HIF. Science 294, 1337-1340 (2001).

20. Epstein, A. C. et al. C. elegans EGL-9 and mammalian homologs define a family of dioxygenases that regulate HIF by prolyl hydroxylation. Cell 107 43-54 (2001).

21. Ivan, M. et al. HIFalpha targeted for VHL-mediated destruction by proline hydroxylation: implications for $\mathrm{O} 2$ sensing. Science 292, 464-468 (2001).

22. Jaakkola, P. et al. Targeting of HIF-alpha to the von Hippel-Lindau ubiquitylation complex by O2-regulated prolyl hydroxylation. Science $\mathbf{2 9 2}, \mathbf{4 6 8 - 4 7 2}$ (2001).

23. Masson, N., Willam, C., Maxwell, P. H., Pugh, C. W. \& Ratcliffe, P. J. Independent function of two destruction domains in hypoxia-inducible factor-alpha chains activated by prolyl hydroxylation. EMBO J. 20, 5197-5206 (2001).

24. Lee, J. W., Bae, S. H., Jeong, J. W., Kim, S. H. \& Kim, K. W. Hypoxia-inducible factor (HIF-1)alpha: its protein stability and biological functions. Exp. Mol. Med. 36, 1-12 (2004).

25. Yee Koh, M., Spivak-Kroizman, T. R. \& Powis, G. HIF-1 regulation: not so easy come, easy go. Trends Biochem. Sci. 33, 526-534 (2008).

26. Taylor, C. T., Doherty, G., Fallon, P. G. \& Cummins, E. P. Hypoxia-dependent regulation of inflammatory pathways in immune cells. J. Clin. Invest. 126 3716-3724 (2016).

27. Semenza, G. L. HIF-1 and tumor progression: pathophysiology and therapeutics. Trends Mol. Med. 8, S62-S67 (2002)

28. Masoud, G. N. \& Li, W. HIF-1alpha pathway: role, regulation and intervention for cancer therapy. Acta Pharm. Sin. B 5, 378-389 (2015)

29. Munkacsy, G. et al. Validation of RNAi silencing efficiency using gene array data shows 18.5\% failure rate across 429 independent experiments. Mol. Ther. Nucleic Acids 5, e366 (2016)

30. Minet, E. et al. HIF1A gene transcription is dependent on a core promoter sequence encompassing activating and inhibiting sequences located upstream from the transcription initiation site and cis elements located within the 5'UTR. Biochem. Biophys. Res. Commun. 261, 534-540 (1999).

31. Ladoux, A. \& Frelin, C. Cardiac expressions of HIF-1 alpha and HLF/EPAS, two basic loop helix/PAS domain transcription factors involved in adaptative responses to hypoxic stresses. Biochem. Biophys. Res. Commun. 240, 552-556 (1997).

32. Danon, A. \& Assouline, G. Antiulcer activity of hypertonic solutions in the rat: possible role of prostaglandins. Eur. J. Pharmacol. 58, 425-431 (1979).

33. Wiener, C. M., Booth, G. \& Semenza, G. L. In vivo expression of mRNAs encoding hypoxia-inducible factor 1. Biochem. Biophys. Res. Commun. 225 485-488 (1996).

34. Palmer, L. A., Semenza, G. L., Stoler, M. H. \& Johns, R. A. Hypoxia induces type II NOS gene expression in pulmonary artery endothelial cells via HIF-1. Am. J. Physiol. 274, L212-L219 (1998).

35. Wenger, R. H., Kvietikova, I., Rolfs, A., Gassmann, M. \& Marti, H. H. Hypoxiainducible factor-1 alpha is regulated at the post-mRNA level. Kidney Int. 51, 560-563 (1997).

36. van Uden, P., Kenneth, N. S. \& Rocha, S. Regulation of hypoxia-inducible factor-1alpha by NF-kappaB. Biochem. J. 412, 477-484 (2008).

37. Niu, G. et al. Signal transducer and activator of transcription 3 is required for hypoxia-inducible factor-1alpha RNA expression in both tumor cells and tumor-associated myeloid cells. Mol. Cancer Res. 6, 1099-1105 (2008).

38. Bonello, S. et al. Reactive oxygen species activate the HIF-1alpha promoter via a functional NFkappaB site. Arterioscler. Thromb. Vasc. Biol. 27, 755-761 (2007).

39. Park, E. J. et al. Vanillin suppresses cell motility by inhibiting STAT3-mediated HIF-1alpha mRNA expression in malignant melanoma cells. Int. J. Mol. Sci. 18 pii: E532 (2017)

40. Yeo, E. J. et al. YC-1: a potential anticancer drug targeting hypoxia-inducible factor 1. J. Natl Cancer Inst. 95, 516-525 (2003).

41. Schepens, B., Tinton, S. A., Bruynooghe, Y., Beyaert, R. \& Cornelis, S. The polypyrimidine tract-binding protein stimulates HIF-1alpha IRES-mediated translation during hypoxia. Nucleic Acids Res. 33, 6884-6894 (2005).

42. Galban, S. et al. RNA-binding proteins HuR and PTB promote the translation of hypoxia-inducible factor 1alpha. Mol. Cell. Biol. 28, 93-107 (2008).

43. Semenza, G. L. Hydroxylation of HIF-1: oxygen sensing at the molecular level. Physiology 19, 176-182 (2004).

44. Xiao, H., Gu, Z., Wang, G. \& Zhao, T. The possible mechanisms underlying the impairment of HIF-1alpha pathway signaling in hyperglycemia and the beneficial effects of certain therapies. Int. J. Med. Sci. 10, 1412-1421 (2013).
45. Rovetta, F. et al. Cobalt triggers necrotic cell death and atrophy in skeletal C2C12 myotubes. Toxicol. Appl. Pharmacol. 271, 196-205 (2013).

46. Cervellati, F. et al. Hypoxia induces cell damage via oxidative stress in retinal epithelial cells. Free Radic. Res. 48, 303-312 (2014).

47. Yuan, Y., Hilliard, G., Ferguson, T. \& Millhorn, D. E. Cobalt inhibits the interaction between hypoxia-inducible factor-alpha and von Hippel-Lindau protein by direct binding to hypoxia-inducible factor-alpha. J. Biol. Chem. 278, 15911-15916 (2003).

48. Hon, W. C. et al. Structural basis for the recognition of hydroxyproline in HIF-1 alpha by pVHL. Nature 417, 975-978 (2002).

49. Min, J. H. et al. Structure of an HIF-1alpha -pVHL complex: hydroxyproline recognition in signaling. Science 296, 1886-1889 (2002)

50. Jewell, U. R. et al. Induction of HIF-1alpha in response to hypoxia is instantaneous. FASEB J. 15, 1312-1314 (2001).

51. Jeong, J. W. et al. Regulation and destabilization of HIF-1alpha by ARD1mediated acetylation. Cell 111, 709-720 (2002).

52. Cheng, J., Kang, X., Zhang, S. \& Yeh, E. T. SUMO-specific protease 1 is essential for stabilization of HIF1alpha during hypoxia. Cell 131, 584-595 (2007).

53. Carbia-Nagashima, A. et al. RSUME, a small RWD-containing protein, enhances SUMO conjugation and stabilizes HIF-1alpha during hypoxia. Cell 131, 309-323 (2007).

54. Liu, Y. V. et al. Calcineurin promotes hypoxia-inducible factor 1alpha expression by dephosphorylating RACK1 and blocking RACK1 dimerization. J. Biol. Chem. 282, 37064-37073 (2007).

55. Baek, J. H. et al. Spermidine/spermine N(1)-acetyltransferase-1 binds to hypoxia-inducible factor-1alpha (HIF-1alpha) and RACK1 and promotes ubiquitination and degradation of HIF-1alpha. J. Biol. Chem. 282 33358-33366 (2007).

56. Mottet, D. et al. Regulation of hypoxia-inducible factor-1alpha protein level during hypoxic conditions by the phosphatidylinositol 3-kinase/Akt/glycogen synthase kinase 3beta pathway in HepG2 cells. J. Biol. Chem. 278, 31277-31285 (2003).

57. Pugh, C. W. \& Ratcliffe, P. J. The von Hippel-Lindau tumor suppressor, hypoxia-inducible factor-1 (HIF-1) degradation, and cancer pathogenesis. Semin. Cancer Biol. 13, 83-89 (2003).

58. Kaelin, W. G. Von Hippel-Lindau disease. Annu. Rev. Pathol. 2, 145-173 (2007).

59. Maynard, M. A. \& Ohh, M. Von Hippel-Lindau tumor suppressor protein and hypoxia-inducible factor in kidney cancer. Am. J. Nephrol. 24, 1-13 (2004).

60. Gnarra, J. R. et al. Mutations of the VHL tumour suppressor gene in renal carcinoma. Nat. Genet. 7, 85-90 (1994)

61. Cancer Genome Atlas Research, N. Comprehensive molecular characterization of clear cell renal cell carcinoma. Nature 499, 43-49 (2013).

62. Herman, J. G. et al. Silencing of the VHL tumor-suppressor gene by DNA methylation in renal carcinoma. Proc. Natl Acad. Sci. USA 91, 9700-9704 (1994).

63. Iliopoulos, O., Kibel, A., Gray, S. \& Kaelin, W. G. Jr. Tumour suppression by the human von Hippel-Lindau gene product. Nat. Med. 1, 822-826 (1995).

64. Schodel, J. et al. Hypoxia, hypoxia-inducible transcription factors, and renal cancer. Eur. Urol. 69, 646-657 (2016)

65. Jung, C. R. et al. E2-EPF UCP targets $\mathrm{PVHL}$ for degradation and associates with tumor growth and metastasis. Nat. Med. 12, 809-816 (2006).

66. Pezzuto A. \& Carico E. Role of HIF-1 in cancer progression: novel insights: a review. Curr. Mol. Med. 18, 343-351 (2018).

67. Ryan, S., Taylor, C. T. \& McNicholas, W. T. Selective activation of inflammatory pathways by intermittent hypoxia in obstructive sleep apnea syndrome. Circulation 112, 2660-2667 (2005).

68. Taylor, C. T., Kent, B. D., Crinion, S. J., McNicholas, W. T. \& Ryan, S. Human adipocytes are highly sensitive to intermittent hypoxia induced NF-kappaB activity and subsequent inflammatory gene expression. Biochem. Biophys. Res. Commun. 447, 660-665 (2014).

69. Prabhakar, N. R. \& Semenza, G. L. Adaptive and maladaptive cardiorespiratory responses to continuous and intermittent hypoxia mediated by hypoxiainducible factors 1 and 2. Physiol. Rev. 92, 967-1003 (2012).

70. Nanduri, J. et al. HIF-1alpha activation by intermittent hypoxia requires NADPH oxidase stimulation by xanthine oxidase. PLOS ONE 10, e0119762 (2015)

71. Lee, H. C. \& Wei, Y. H. Mitochondria and aging. Adv. Exp. Med. Biol. 942, 311-327 (2012).

72. Bereiter-Hahn, J. Do we age because we have mitochondria? Protoplasma 251, 3-23 (2014) 
73. Yuan, $Y$. et al. Regulation of SIRT1 in aging: roles in mitochondrial function and biogenesis. Mech. Ageing Dev. 155, 10-21 (2016).

74. Morris, B. J. Seven sirtuins for seven deadly diseases of aging. Free Radic. Biol. Med. 56, 133-171 (2013).

75. Sasaki, T., Maier, B., Bartke, A. \& Scrable, H. Progressive loss of SIRT1 with cell cycle withdrawal. Aging Cell. 5, 413-422 (2006).

76. Okazaki, M. et al. PPARbeta/delta regulates the human SIRT1 gene transcription via Sp1. Endocr. J. 57, 403-413 (2010).

77. Li, N., Muthusamy, S., Liang, R., Sarojini, H. \& Wang, E. Increased expression of miR-34a and miR-93 in rat liver during aging, and their impact on the expression of Mgst1 and Sirt1. Mech. Ageing Dev. 132, 75-85 (2011).

78. Dong, Z. X. et al. (-)-epicatechin suppresses angiotensin ii-induced cardiac hypertrophy via the activation of the SP1/SIRT1 signaling pathway. Cell. Physiol. Biochem. 41, 2004-2015 (2017).

79. Gomes, A. P. et al. Declining NAD(+) induces a pseudohypoxic state disrupting nuclear-mitochondrial communication during aging. Cell 155, 1624-1638 (2013).

80. Wenz, T. Regulation of mitochondrial biogenesis and PGC-1alpha under cellular stress. Mitochondrion 13, 134-142 (2013).

81. Chung, S. et al. Regulation of SIRT1 in cellular functions: role of polyphenols. Arch. Biochem. Biophys. 501, 79-90 (2010).

82. LaRocca, T. J., Hearon, C. M. Jr., Henson, G. D. \& Seals, D. R. Mitochondrial quality control and age-associated arterial stiffening. Exp. Gerontol. 58, 78-82 (2014).

83. Revollo, J. R. \& Li, X. The ways and means that fine tune Sirt1 activity. Trends Biochem. Sci. 38, 160-167 (2013).

84. Satoh, A. et al. Sirt1 extends life span and delays aging in mice through the regulation of Nk2 homeobox 1 in the DMH and LH. Cell. Metab. 18, 416-430 (2013).

85. Mendelsohn, A. R. \& Larrick, J. W. Partial reversal of skeletal muscle aging by restoration of normal $\mathrm{NAD}(+)$ levels. Rejuvenation Res. 17, 62-69 (2014).

86. Brenmoehl, J. \& Hoeflich, A. Dual control of mitochondrial biogenesis by sirtuin 1 and sirtuin 3. Mitochondrion 13, 755-761 (2013).

87. Gowans, G. J., Hawley, S. A., Ross, F. A. \& Hardie, D. G. AMP is a true physiological regulator of AMP-activated protein kinase by both allosteric activation and enhancing net phosphorylation. Cell. Metab. 18, 556-566 (2013).

88. Hardie, D. G., Ross, F. A. \& Hawley, S. A. AMPK: a nutrient and energy sensor that maintains energy homeostasis. Nat. Rev. Mol. Cell Biol. 13, 251-262 (2012).

89. Salminen, A., Kaarniranta, K. \& Kauppinen, A. Age-related changes in AMPK activation: Role for AMPK phosphatases and inhibitory phosphorylation by upstream signaling pathways. Ageing Res. Rev. 28, 15-26 (2016).

90. Burkewitz, K., Zhang, Y. \& Mair, W. B. AMPK at the nexus of energetics and aging. Cell. Metab. 20, 10-25 (2014).

91. Salminen, A. \& Kaarniranta, K. AMP-activated protein kinase (AMPK) controls the aging process via an integrated signaling network. Ageing Res. Rev. 11, 230-241 (2012).

92. Wang, Y., Zhao, X., Lotz, M., Terkeltaub, R. \& Liu-Bryan, R. Mitochondrial biogenesis is impaired in osteoarthritis chondrocytes but reversible via peroxisome proliferator-activated receptor gamma coactivator 1alpha. Arthritis Rheumatol. 67, 2141-2153 (2015).

93. Shaw, R. J. et al. The tumor suppressor LKB1 kinase directly activates AMPactivated kinase and regulates apoptosis in response to energy stress. Proc. Natl Acad. Sci. USA 101, 3329-3335 (2004).

94. Jones, R. G. et al. AMP-activated protein kinase induces a p53-dependent metabolic checkpoint. Mol. Cell 18, 283-293 (2005).

95. Lee, C. W. et al. AMPK promotes p53 acetylation via phosphorylation and inactivation of SIRT1 in liver cancer cells. Cancer Res. 72, 4394-4404 (2012).

96. Okoshi, R. et al. Activation of AMP-activated protein kinase induces p53dependent apoptotic cell death in response to energetic stress. J. Biol. Chem. 283, 3979-3987 (2008).

97. Jager, S., Handschin, C., St-Pierre, J. \& Spiegelman, B. M. AMP-activated protein kinase (AMPK) action in skeletal muscle via direct phosphorylation of PGC1alpha. Proc. Natl Acad. Sci. USA 104, 12017-12022 (2007).

98. Amat, R. et al. SIRT1 controls the transcription of the peroxisome proliferatoractivated receptor-gamma Co-activator-1alpha (PGC-1alpha) gene in skeletal muscle through the PGC-1alpha autoregulatory loop and interaction with MyoD. J. Biol. Chem. 284, 21872-21880 (2009).

99. Rodgers, J. T., Lerin, C., Gerhart-Hines, Z. \& Puigserver, P. Metabolic adaptations through the PGC-1 alpha and SIRT1 pathways. FEBS Lett. 582, 46-53 (2008).
100. Gui, D. et al. Salidroside attenuates hypoxia-induced pulmonary arterial smooth muscle cell proliferation and apoptosis resistance by upregulating autophagy through the AMPK-mTOR-ULK1 pathway. BMC Pulm. Med. 17, 191 (2017).

101. Johnson, S. C., Rabinovitch, P. S. \& Kaeberlein, M. mTOR is a key modulator of ageing and age-related disease. Nature 493, 338-345 (2013).

102. Zoncu, R., Efeyan, A. \& Sabatini, D. M. mTOR: from growth signal integration to cancer, diabetes and ageing. Nat. Rev. Mol. Cell Biol. 12, 21-35 (2011).

103. Lauring, J., Park, B. H. \& Wolff, A. C. The phosphoinositide-3-kinase-Akt-mTOR pathway as a therapeutic target in breast cancer. J. Natl Compr. Canc. Netw. 11, 670-678 (2013)

104. Wang, J., Zhong, C., Wang, F., Qu, F. \& Ding, J. Crystal structures of S6K1 provide insights into the regulation mechanism of S6K1 by the hydrophobic motif. Biochem. J. 454, 39-47 (2013)

105. Hahn-Windgassen, A. et al. Akt activates the mammalian target of rapamycin by regulating cellular ATP level and AMPK activity. J. Biol. Chem. 280, 32081-32089 (2005)

106. Inoki, K., Zhu, T. \& Guan, K. L. TSC2 mediates cellular energy response to control cell growth and survival. Cell 115, 577-590 (2003).

107. Kim, J., Kundu, M., Viollet, B. \& Guan, K. L. AMPK and mTOR regulate autophagy through direct phosphorylation of Ulk1. Nat. Cell Biol. 13, 132-141 (2011).

108. Egan, D. F. et al. Phosphorylation of ULK1 (hATG1) by AMP-activated protein kinase connects energy sensing to mitophagy. Science $\mathbf{3 3 1}$ 456-461 (2011)

109. Inoki, K., Li, Y., Xu, T. \& Guan, K. L. Rheb GTPase is a direct target of TSC2 GAP activity and regulates mTOR signaling. Genes Dev. 17, 1829-1834 (2003).

110. Gwinn, D. M. et al. AMPK phosphorylation of raptor mediates a metabolic checkpoint. Mol. Cell 30, 214-226 (2008).

111. Mizushima, N. The role of the Atg1/ULK1 complex in autophagy regulation. Curr. Opin. Cell Biol. 22, 132-139 (2010).

112. Shang, L. et al. Nutrient starvation elicits an acute autophagic response mediated by Ulk1 dephosphorylation and its subsequent dissociation from AMPK. Proc. Natl Acad. Sci. USA 108, 4788-4793 (2011).

113. Salminen, A., Kaarniranta, K. \& Kauppinen, A. AMPK and HIF signaling pathways regulate both longevity and cancer growth: the good news and the bad news about survival mechanisms. Biogerontology 17, 655-680 (2016)

114. Gray, M. J. et al. HIF-1alpha, STAT3, CBP/p300 and Ref-1/APE are components of a transcriptional complex that regulates Src-dependent hypoxia-induced expression of VEGF in pancreatic and prostate carcinomas. Oncogene 24, 3110-3120 (2005).

115. Salminen, A., Hyttinen, J. M. \& Kaarniranta, K. AMP-activated protein kinase inhibits NF-kappaB signaling and inflammation: impact on healthspan and lifespan. J. Mol. Med. 89, 667-676 (2011).

116. Shackelford, D. B. \& Shaw, R. J. The LKB1-AMPK pathway: metabolism and growth control in tumour suppression. Nat. Rev. Cancer 9, 563-575 (2009).

117. Luo, Z., Zang, M. \& Guo, W. AMPK as a metabolic tumor suppressor: control of metabolism and cell growth. Future Oncol. 6, 457-470 (2010).

118. Ikhlas, S. \& Ahmad, M. Metformin: Insights into its anticancer potential with special reference to AMPK dependent and independent pathways. Life. Sci. 185, 53-62 (2017).

119. Faa, G. et al. Fetal programming of the human brain: is there a link with insurgence of neurodegenerative disorders in adulthood? Curr. Med. Chem 21, 3854-3876 (2014).

120. Nalivaeva, N. N., Turner, A. J. \& Zhuravin, I. A. Role of prenatal hypoxia in brain development, cognitive functions, and neurodegeneration. Front. Neurosci. 12, 825 (2018)

121. Erecinska, M. \& Silver, I. A. Tissue oxygen tension and brain sensitivity to hypoxia. Respir. Physiol. 128, 263-276 (2001).

122. Oechmichen, M. \& Meissner, C. Cerebral hypoxia and ischemia: the forensic point of view: a review. J. Forensic Sci. 51, 880-887 (2006).

123. Vasilev, D. S., Dubrovskaya, N. M., Tumanova, N. L. \& Zhuravin, I. A. Prenatal hypoxia in different periods of embryogenesis differentially affects cell migration, neuronal plasticity, and rat behavior in postnatal ontogenesis. Front. Neurosci. 10, 126 (2016).

124. Gross, J., Burgoyne, R. D. \& Rose, S. P. Influence of prenatal hypoxia on brain development: effects on body weight, brain weight, DNA, protein, acetylcholinesterase, 3-quinuclidinyl benzilate binding, and in vivo incorporation of [14C]lysine into subcellular fractions. J. Neurochem. 37, 229-237 (1981). 
125. Lahiri, D. K., Ghosh, C. \& Ge, Y. W. A proximal gene promoter region for the beta-amyloid precursor protein provides a link between development, apoptosis, and Alzheimer's disease. Ann. N. Y. Acad. Sci. 1010 643-647 (2003).

126. Peyronnet, J. et al. Developmental plasticity of the carotid chemoafferent pathway in rats that are hypoxic during the prenatal period. Eur. J. Neurosci. 26, 2865-2872 (2007)

127. Kerridge, C., Kozlova, D. I., Nalivaeva, N. N. \& Turner, A. J. Hypoxia affects neprilysin expression through caspase activation and an APP intracellular domain-dependent mechanism. Front. Neurosci. 9, 426 (2015).

128. Nanduri, J. \& Prabhakar, N. R. Epigenetic regulation of carotid body oxygen sensing: clinical implications. Adv. Exp. Med. Biol. 860, 1-8 (2015).

129. Chiang, A. A. Obstructive sleep apnea and chronic intermittent hypoxia: a review. Chin. J. Physiol. 49, 234-243 (2006).

130. Shamsuzzaman, A. S., Gersh, B. J. \& Somers, V. K. Obstructive sleep apnea: implications for cardiac and vascular disease. JAMA 290, 1906-1914 (2003).
131. Lavie, L. Oxidative stress in obstructive sleep apnea and intermittent hypoxia--revisited--the bad ugly and good: implications to the heart and brain. Sleep Med. Rev. 20, 27-45 (2015).

132. Drager, L. F. et al. The impact of obstructive sleep apnea on metabolic and inflammatory markers in consecutive patients with metabolic syndrome. Plos ONE 5, e12065 (2010).

133. Douglas, N. et al. Prevalence of depression in patients referred with snoring and obstructive sleep apnoea. Intern. Med. J. 43, 630-634 (2013).

134. Rosenzweig, I. et al. Sleep apnoea and the brain: a complex relationship. Lancet Respir. Med. 3, 404-414 (2015).

135. Gozal, D., Ham, S. A. \& Mokhlesi, B. Sleep apnea and cancer: analysis of a nationwide population sample. Sleep 39, 1493-1500 (2016).

136. Gaspar, L. S., Alvaro, A. R., Moita, J. \& Cavadas, C. Obstructive sleep apnea and hallmarks of aging. Trends Mol. Med. 23, 675-692 (2017).

137. Kukwa, W., Migacz, E., Druc, K., Grzesiuk, E. \& Czarnecka, A. M. Obstructive sleep apnea and cancer: effects of intermittent hypoxia? Future Oncol. 11, 3285-3298 (2015). 